\title{
Medicalización de la Vida. Abordaje de su Demanda en Salud Mental
}

\section{Medicalization of Life. Boarding its Demand in Mental Health}

\author{
Miguel A. Moré Herrero \\ Ámbito privado, España
}

\begin{abstract}
Resumen. El fenómeno de la medicalización/psicopatologización de la vida va en aumento en los países desarrollados, por la coincidencia de intereses de diversos agentes en ello, y los conceptos cambiantes del binomio salud/enfermedad, existiendo controversias que derivan de la tipificación enfermedad/no enfermedad y acerca de la indicación o no de tratamiento. En última instancia, es el profesional sanitario (médico/psiquiatra/psicólogo) el que lo sanciona, pero debe tenerse en cuenta que su morbilidad y su impacto social y económico son muy importantes.

La repercusión de esta demanda en el ámbito asistencial público ha ido creciendo exponencialmente, desbordando la capacidad de respuesta de los equipos y siendo controvertidos los resultados de su intervención, lo que exige que los servicios públicos mejoren su coordinación, organización y la formación de sus profesionales para que puedan intervenir de forma más eficiente y eficaz tanto en su abordaje asistencial, como en una indicación de no tratamiento aceptable para el usuario.

Palabras clave: medicalización de la vida, demanda, abordaje, servicios públicos.
\end{abstract}

\begin{abstract}
The phenomenon of life's medicalization is increasing in developed countries, where several agent's interests and changing concepts on health/disease meet. The existing controversies lead to the typification of pathology/non pathology, or the need for treatment/non treatment, leaving to the health specialist (doctor/psychiatrist/psychologist) the final decision. It must be taking into consideration the importance of its morbility as well as its social and economic impact.

The consequences of this specific demand in the public health system have grown exponentially, sometimes overwhelming the capacity of teams to meet these needs, while at the same time getting controversial results from their interventions. This might mean that public services need to improve the coordination, organization and training of the professional staff in order to become more efficient both in assisting, as well as in determining in an acceptable manner when treatment is not necessary.
\end{abstract}

Keywords: medicalization of life, demand, aboard, public services.

\section{Introducción}

El fenómeno de la medicalización (o en el ámbito de la salud mental, psicopatologización) de la vida, se ha ido desarrollando progresivamente y en nuestra cultura y sociedad actuales, alcanza una dimensión cuya reper-

Miguel A. Moré Herrero es Psiquiatra y ha sido Jefe del Servicio de Salud Mental de Villaverde (Madrid) desde 1990 hasta 2010.

La correspondencia sobre este artículo debe enviarse al e-mail: miguelmore@yahoo.com 
cusión en el consumo y gasto de los servicios sanitarios exige propuestas que permitan una mejora de su gestión. Se ha definido la medicalización (Kishore, 2002), como "la forma en que el ámbito de la medicina moderna se ha expandido en los años recientes y ahora abarca muchos problemas que antes no estaban considerados como entidades médicas".

La medicalización (Mintzes, 2002) es un proceso por el que problemas no médicos se tratan como si lo fueran, normalmente en términos de enfermedad o trastorno, o dicho de otra manera, medicalizar la vida es convertir un problema personal o social en un problema médico susceptible de ser abordado por profesionales de la salud.

Ya anteriormente (Meador, 1965) se definía la "no enfermedad como aquel problema humano definido en alguna instancia (social o sanitaria) como condición médica, para el que se obtendría mejores resultados si no fuera considerado como tal".

La medicalización de la vida es un proceso, generalmente propio de los países desarrollados, que se va desarrollando paulatinamente, cuando se dan las siguientes condiciones necesarias (Orueta Sánchez, 2011):

a) La significación como anormal por parte de sectores poderosos de la sociedad de una situación o sufrimiento que antes no se consideraba como tal, convirtiéndolo en un "problema" para el que no hay soluciones actuales y hay que crearlas, ofreciéndose algunos de estos colectivos (industria farmacéutica) a ello.

b) Los distintos actores implicados en el proceso de asistencia sanitaria deben aceptar que dicha situación forma parte de su campo de actuación, lo cual se ve influido por presiones externas (sociales, de la industria, etc.).

c) El sector sanitario busca soluciones para dar respuesta a los nuevos retos, lo que requiere la puesta en marcha de pruebas diagnósticas y tratamientos, produciéndose el fenómeno de la medicalización.

d) Con frecuencia el círculo se cierra por la insuficiente respuesta conseguida, que genera nueva inseguridad y necesidad de nuevas actuaciones que el sector sanitario asume.

En los países desarrollados la sociedad ha desplazado al campo médico la búsqueda de soluciones a problemas inherentes a las circunstancias de la vida y así,"la obsesión por una salud perfecta se ha convertido en el factor patógeno predominante" (Illich, 1999), con lo que "una persona sana es solamente un enfermo sin identificar", produciéndose una paradoja de la salud, consistente en que cuanto mayor es la situación objetiva de salud, mayores son los problemas de salud declarados y cuantos más recursos se emplean en la salud, mayor es la percepción de estar enfermos.

Situaciones de la vida como el cansancio o la frustración o variantes de la normalidad como la calvicie o la fealdad se están considerando enfermedades y esto se extiende a etapas de la vida como el nacimiento, adolescencia, menopausia, etc.

En estos momentos, el derecho a la salud se está convirtiendo en un peligroso objeto de consumo, con una dependencia de la medicina que incapacita a las personas para cuidarse a sí mismas y un baja tolerancia a la frustración y al sufrimiento.

En esta línea de una tendencia en aumento a clasificar como enfermedades los problemas de la gente, se cita el resultado de una encuesta (Smith, 2002) que el British Medical Journal propuso a sus lectores sobre cuáles son en nuestro entorno las principales no-enfermedades, siendo en orden descendente: envejecimiento, trabajo, aburrimiento, bolsas en los ojos, ignorancia, calvicie, pecas, orejas grandes, canas, fealdad, parto, alergia al siglo XXI, jet lag, infelicidad, celulitis, resaca, ansiedad por el tamaño del pene, embarazo, cabreo al volante y soledad.

La concepción de las relaciones cambiantes entre salud y enfermedad está en la base de este problema (Márquez y Meneu, 2003) y así frente a un concepto objetivo de salud, existe un concepto subjetivo de ella, pudiéndose decir que tanto el concepto de salud como el de enfermedad son constructos sociales.

Evidentemente, que la definición que la Organización Mundial de la Salud (OMS, 1948) propugna de la salud como "el completo bienestar físico, psicológico y social", propiciaría el problema de la medicalización de la vida, ya que en dicho concepto parece que cualquier tipo de sufrimiento es enfermedad, y que la salud ha dejado de ser (como etimológicamente es) la capacidad de superar los avatares de la vida diaria para pasar a ser 
la ausencia de todo tipo de problemas, lo que ha dado pie a que un autor como Smith (2002) cite a Loeffler, cuando ironiza sobre el concepto de salud de la OMS, diciendo que " dicho estado solo se alcanza en el punto del orgasmo simultáneo, dejándonos a la mayoría de la población enferma durante la mayor parte del tiempo".

La distinción entre lo normal y lo patológico, entre lo que es o no enfermedad, es coyuntural y depende del significado que le es atribuido en distintas épocas y entre distintas culturas (Ortíz Lobo, 2008; Fernández Liria, 2003). En nuestra cultura actual, cada día más, la definición de enfermedad va ligada a las disponibilidades tecnológicas y también, cada vez más, se define la enfermedad ante simples síntomas o signos, aspectos estéticos, presencia de factores de riesgo o por la probabilidad de padecer en el futuro una enfermedad (Marquez y Meneu, 2003).

En un editorial del Osakidetza (2005) se recoge cómo la idea de que prevenir es mejor que curar ha dado lugar en esta sociedad a que el riesgo haya pasado a ser considerado una enfermedad prevalente, y aún siendo la prevención un aspecto importante de la actuación terapéutica, sin embargo, se debe tener en cuenta que la sobreprevención puede crear iatrogenia, tanto por las actividades diagnósticas como por la instauración de tratamientos (Gervas y Pérez Fernández, 2003), motivando la necesidad de una prevención cuaternaria que trate de evitar o atenuar estas sobre intervenciones (Ortíz Lobo e Ibáñez Rojo, 2012).

Se hace necesario pues, encontrar un equilibrio entre la prevención de riesgos y la iatrogenia que esto puede causar, porque como se recoge (Ebraim, 2002), citando a Skrabanek, "puesto que la vida es una enfermedad universal de transmisión sexual y mortal de necesidad, vivirla con plenitud requiere un equilibro entre el riesgo razonable y el no razonable".

Todo ello, contribuye a una expansión terapéutica que se traduce en que:

- Se considera necesario el control médico de etapas de la vida (nacimiento, adolescencia, menopausia).

- Problemas personales/sociales pasan a considerarse como problemas médicos (tristeza, duelo, síndrome postvacacional, desorden de deficiencia motivacional o pereza).

- Factores de riesgo pasan a considerarse como auténticas enfermedades (dislipemia, osteoporosis, etc.).

- Situaciones o cuadros poco frecuentes pasan a considerarse como muy frecuentes (disfunción eréctil, disfunción sexual femenina, etc.).

- Síntomas o cuadros leves pasan a considerarse como graves (colon irritable, síndrome premenstrual).

Se han señalado (Marquez y Meneu, 2003) una serie de protagonistas de esta medicalización, advirtiendo que los factores que contribuyen al fenómeno son diversos y complejos y que en este proceso las interacciones y sinergias son múltiples, pero se coincide en que a la postre (Ortíz Lobo, 2008), los médicos (u otros profesionales sanitarios como los psicólogos), son los principales agentes de ella, porque "en última instancia es en la consulta de los servicios sanitarios donde los profesionales sancionan qué es enfermedad y qué no".

Se han mencionado (Orueta et al., 2011) toda una serie de factores causales en el fenómeno de la medicalización, enmarcados en 5 grandes grupos: sociedad, medios de comunicación, instituciones político-sanitarias, profesionales e industria farmacéutica, que de forma sucinta paso a recoger:

\section{Sociedad}

- Cambio en el concepto de salud/enfermedad.

- Aumento del valor de la salud en la sociedad (el miedo al sufrimiento y la enfermedad contribuye a este aumento y al final "la sociedad está enfermando por no enfermar").

- Mayor acceso a la información, aunque de características parciales e incompletas (utilizada por la industria con un gasto del $20 \%$ en publicidad en forma de "publireportajes", "campañas de concienciación”, etc., que pueden tener características maliciosas).

- Secularización de la sociedad (declive de la función religiosa en el consuelo del sufrimiento).

- Medicina/Psicología como parte del mercado de consumo.

- Fascinación por la tecnología (sobrevaloración de las pruebas frente a la relación médico-paciente). 
- Expectativas ilimitadas (se crean expectativas irreales y se espera que la medicina/psicología lo resuelva todo).

- Disminución de la tolerancia al dolor y sufrimiento (hasta la tolerancia cero).

- Búsqueda de soluciones inmediatas.

- Disminución de la capacidad de autocuidados.

- Reducción de las redes informales de cuidado.

- Escasa educación sanitaria de la población.

\section{Medios de comunicación}

- Fuente de información sanitaria.

- Poder mediático.

- Sesgos de información.

- Información versus publicidad.

\section{Instituciones político-sanitarias}

- Modelo universal, accesible y abierto (indefinición de cartera de servicios).

- Modelo basado en demandas y no en necesidades.

- Políticas paternalistas y acríticas.

- Papel preponderante de la atención hospitalaria.

- Creación de estructuras/sistemas paralelos.

- Burocratización de la asistencia.

- Expansión acrítica de la medicina preventiva.

\section{Profesionales}

- Grupo fundamental en la medicalización.

- Pérdida de la visión multifactorial de la enfermedad (biologismo).

- Parcelación de la atención. (superespecialización).

- Sobrecarga de trabajo.

- Trabajo con incertidumbre en Atención Primaria por tratar los primeros síntomas, tendiendo ante la duda y ansiedad a tratar con fármacos desde el principio.

- Dificultad de diferenciación entre normalidad/anormalidad.

- Conversión a enfermedad de los factores de riesgo.

- Tendencia a la innovación científica.

- Sesgos en el rigor científico de los estudios.

- Relaciones con la industria farmacéutica.

\section{Industria farmacéutica}

- Como empresa busca obtener beneficios.

- Búsqueda de mercados.

- Información sesgada.

- Promoción de enfermedades (TDAH, Trastorno de Ansiedad social).

- Promoción de medicamentos. 


\section{Psicopatologización de los avatares de la vida}

El fenómeno en general de la medicalización de la vida tiene una repercusión especial en el ámbito de la salud mental, y su correlato es la psiquiatrización y/o psicologización de los avatares de la vida y la incidencia en su manejo asistencial.

Soportar la frustración, cuando esta es inevitable, soportar la pena y la tristeza que nos abaten (que no deprimen), soportar y sentir las pérdidas, ha llegado a ser cada vez más ocasional, escondido, marginalizado y estigmatizado y cuando esto se medicaliza, se recurre a un tratamiento indiscriminado mediante psicofármacos y/o psicoterapia. Esto tiene su origen en una ideología social, una cultura, que, cada vez más, resulta intolerante e incapaz de soportar las pérdidas y las frustraciones afectivas y vitales, fruto de nuestra intolerancia al sufrimiento y la ansiedad, y así se señala (Tizón 2004) que, hablar, esperar y observar, es algo que le resulta difícil de aceptar al consultante y al médico o al psicólogo. A aquel por su tendencia a asumir un rol pasivo y supuestamente falto de recursos, $\mathrm{y}$ a estos por una tendencia a pretender tener una respuesta omnipotente para todo.

Pero no son solo los potenciales pacientes o usuarios los que demandan atención, sino que nuevas profesiones y profesionales se han venido a sumar a la tarea y reclaman sus correspondientes ámbitos de trabajo, y por otra parte, los servicios sanitarios, públicos o privados, se ven en la necesidad de ofertar nuevas formas de asistencia y así, las prácticas psicoterapéuticas y el derecho a disponer de ellas se han extendido a amplios sectores sociales que reclaman ayuda profesional para tratar de afrontar sus problemas vitales y encarar mejor la difícil conquista de la felicidad (Retolaza, 2008).

Junto lo anterior, y no en menor importancia, los poderes públicos contribuyen a esta psicopatologización de lo cotidiano al trasladar al ámbito individual problemas sociales, y convertir la causa y solución de estos en un problema individual para el que a su vez los sujetos buscan respuesta en los servicios sanitarios.

Sin embargo, si entendemos que no todo sufrimiento es enfermedad, tendremos que delimitar lo mejor posible qué tratar y qué no y esto a su vez estará en función del ámbito asistencial, público o privado, en el que se realice la intervención. Aunque en el ámbito privado la opinión del paciente es fundamental en la indicación de tratamiento, siempre debe haber una actitud ética del profesional en su abordaje (Ortíz Lobo, 2008).

Pero es en el ámbito de lo público, cuando nuestra condición de terapeutas y a la vez gestores de los recursos sanitarios nos exige discriminar lo adecuado o no, y lo potencialmente beneficioso o no de nuestras intervenciones.

La función encomendada a los dispositivos de salud mental públicos por la Ley General de Sanidad de 1986 (Ministerio de Sanidad y Consumo, 1986) suponía atender a una población circunscrita en las mejores condiciones posibles de equidad, accesibilidad, disponibilidad y continuidad de cuidados.

Hoy día, las políticas revisionistas y de recortes están condicionando seriamente esta función, pero en cualquier caso y desde una concepción comunitaria asistencial, resulta necesaria una identificación de las necesidades asistenciales de la población para una mejor proporcionalidad asistencial.

El término necesidad es ambiguo conceptualmente y viene definido socialmente, pudiendo ser las necesidades de distinta índole (normativas, sentidas, expresadas o comparativas) (Royse, 1982). Sin embargo, puede haber o no una percepción de dichas necesidades, de lo que se va a derivar o no una demanda de cuidados y esta a su vez producirá una utilización de los servicios (Asociación Española de Neuropsiquiatría AEN,1998).

Por otra parte, las prestaciones asistenciales de un servicio público de salud mental no deben tener en cuenta solo la demanda expresada, sino que deben atender a necesidades existentes y no expresadas (patologías graves que no acuden, especiales grupos de riesgo de la población, etc.).

Esto supone, en la práctica asistencial, como recogía el grupo de estudio ya citado (AEN, 1998), establecer unas prioridades y hacer ofertas asistenciales proporcionales a la gravedad de las demandas, ya que es un hecho la saturación que los servicios de salud mental tienen por problemas menores, que aunque generan sufrimiento, desbordan su capacidad asistencial, siendo difícil mantener respuestas adecuadas tanto a los casos más graves como a este otro tipo de demandas (Royse, 1982; Harrison, 2000), máxime dada la alta percepción de nece- 
sidad de ayuda que tienen los pacientes con patologías más leves en detrimento de los más graves (Herran et al., 2000; Kessler et al., 1997).

En términos generales, el concepto de salud como bien de consumo en una población cada vez más envejecida y crónica, determina un generalizado aumento de consumo y gasto sanitario, volviéndose escasos los recursos disponibles y necesario el asignarlos racionalmente (Maineti, 2006), y más aún en los dispositivos de salud mental, dada la indefinición de una cartera de servicios que ayudara a esta racionalidad.

Esto ha motivado el interés de establecer perfiles asistenciales (Moré, Jiménez Arriero, Muñoz, Muñoz de Morales y Zufia, 2008) para una mejor discriminación y atención de la demanda, dada la alta prevalencia de morbilidad psíquica en Atención Primaria, que con la inclusión de los trastornos menores alcanza cifras del 4050\% de la demanda (Padierna Acero, Gastain Sáenz, Díaz Lopez y Etxebeste Anton, 1988; Vazquez Barquero, 1990), como ha sido replicado en un reciente trabajo por nosotros mismos (Moré et al., 2008).

\section{Abordaje de la demanda}

Se ha señalado la controversia entre tratar o no estos trastornos menores o malestares (Ortíz Lobo, 2008; Rendueles, 2009) y cómo las intervenciones psicoterapéuticas o psicofarmacólogicas pueden encontrar una justificación en sus potenciales capacidades para lograr una mayor calidad de vida del demandante (Layard, 2006; Charlton, 2003). No obstante, desde los limitados recursos públicos asistenciales, nuestra tarea debe ser discriminar lo más adecuadamente posible la gravedad de las patologías, atendiendo a su diagnóstico, comorbilidad, grado de discapacidad, repercusión en el entorno, tiempo de evolución, pertenencia a grupos o factores de riesgo, etc. (AEN, 1998) y en función de todo ello "sancionar" la existencia o no de enfermedad que precise indicación de tratamiento.

Igualmente, existe un debate sobre el nivel asistencial (Atención Primaria o Atención Especializada) en que deberán ser atendidas estas demandas. Si es evidente que su inicial recepción es en el nivel de la Atención Primaria, se discute sobre si debe ser en este nivel donde se atiendan exclusivamente, siendo el papel de la especializada el de actuar como consultores/asesores (Retolaza, 2008), o lo limitado de los recursos en tiempo y formación de los profesionales de Atención primaria permitiría dicha exclusiva atención en este nivel.

En la práctica, se constata una dificultad para que lo deseable sea plausible y es una realidad también el progresivo abandono de la coordinación entre Atención Primaria y los Servicios de Salud Mental, por lo que sin renunciar a la necesidad de una mejora de esta coordinación, con lo que implica de hacer factibles las tareas de consultoría y asesoramiento mencionadas, es necesario articular "procedimientos que separen las intervenciones posibles de las que no lo son, definan su grado y nivel de alcance necesario, e incluso determinen quién debe o no llevarlas a cabo"(Retolaza, 2008).

Se han propuesto intervenciones en el nivel de la Atención Primaria (Moré, 2009) para el manejo de los trastornos mentales comunes, que implican tareas de contención, desmedicalización de los problemas, potenciación de la red social, higienización de los estilos de vida, etc., así como la utilización de recursos terapéuticos tales como counselling, terapias cognitivas (TRE), terapias de resolución de problemas, grupos psicoeducativos, etc., existiendo ejemplos de su práctica y resultados (Aiarzaguena, 2003).

Comparto las opiniones (Retolaza, 2009; Carmona, Gutiérrez, Maqueda, de Osma y Tejero, 2009; Tizón, San José y Nadal, 1997) de que una mejora de los flujos de formación e información entre los dos niveles asistenciales en el marco de una colaboración y cooperación y un proceso de cuidados escalonados debe guiar el abordaje de estas demandas y tratar de dar respuesta a las preguntas sobre si ¿precisan o no de tratamiento?, ¿quién debe administrarlo?, ¿qué tratamiento es el adecuado? y ¿dónde debe tener lugar?

En virtud de esta graduación y los procedimientos a emplear, los profesionales intervinientes incluirán diferentes profesionales sanitarios de los equipos asistenciales con formación específica, que incluya a psicólogos, enfermería y trabajadores sociales para intervenciones diferenciadas. 
Como ayuda a la valoración y abordaje de estos casos, caracterizados por presentar clínica solapada o subumbral y con frecuentes estresores psicosociales que actúan como desencadenantes o condicionantes de la evolución (Goldberg y Huxley, 1992; Kendrik, 2000), se ha propuesto, para ser llevada a cabo por los médicos de Atención Primaria, una Formulación de casos psiquiátricos en Atención Primaria (de la Mata y Ortíz Lobo, 2009) que incluye un diagnóstico clínico, un diagnóstico situacional, el modelo explicativo del paciente sobre los síntomas, las expectativas y demandas del paciente, un plan terapéutico y la devolución al paciente del plan de tratamiento.

Este plan de tratamiento ha sido detallado (Retolaza, 2009) respecto de los trastornos mentales comunes más frecuentes en Atención Primaria (ansiedad, depresión y abuso de alcohol), con especial atención a la detección, síntomas y cuidados más específicos en cada uno de ellos.

En el nivel especializado de los Servicios de Salud Mental la indicación de tratamiento, incluso en el caso de trastornos adaptativos que cumplan criterios como tales (Morera, 1998) y que por su gravedad lo requieran, plantea, a mi juicio, la necesidad de que los Servicios Públicos mejoren su organización para dar respuesta a estas demandas, que pueden llegar a suponer un $20 \%$ de la demanda total. Esto requiere un proceso de mejora de la asistencia de este tipo de trastornos que permita abordarlos con recursos y técnicas potencialmente más adecuadas y optimizadores, a fin de hacer sostenible la atención a esta demanda y no comprometer la de los casos más graves (Moré, 2007). Se han desarrollado modelos (Consejería de Salud de Andalucía, 2003) que en la práctica real introducen mejoras en la atención a los trastornos mentales comunes, a través de un modelo de cooperación en el que están diferenciados los espacios de trabajo de cada nivel asistencial con criterios consensuados de transferencia de casos, habiendo además un espacio de trabajo común.

De no ser así, tal demanda tiende a producir una disfuncionalidad de los servicios a través del siguiente recorrido: tendencia a una psiquiatrización y psicologización en la sociedad de los acontecimientos adversos de la vida $\rightarrow$ que se convierte en demanda en las consultas de atención primaria, por la mayor necesidad de ayuda que tienen estos casos $\rightarrow$ que no es evaluada suficientemente en estas por falta de tiempo y formación $\rightarrow$ que es derivada a servicios especializados de manera indiscriminada $\rightarrow$ produciendo listas de espera prolongadas $\rightarrow$ que hacen que no puedan ser atendidas paradójicamente con la inmediatez que parecería requerir al ser trastornos adaptativos mayoritariamente $\rightarrow$ ocasionando un porcentaje importantes de ausencias (hasta del 30\%) $\rightarrow$ que deterioran la eficiencia asistencial y aumentan el gasto $\rightarrow$ así como retrasan la intervención en los casos más necesitados $\rightarrow$ o hacen que estas intervenciones no puedan realizarse en condiciones técnicas adecuadas (en términos de frecuencia y plazos de tiempo) que afectan a su calidad y efectividad $\rightarrow$ pudiendo generar insatisfacción en los pacientes y profesionales.

Un proyecto de este tipo (Moré, 2007) implicaría una categorización de la población diana, una cuantificación de su demanda, el establecimiento de criterios de recepción, de un procedimiento de evaluación y un protocolo de intervenciones que contemple el diagnóstico, necesidades asistenciales, objetivos terapéuticos, intervenciones a llevar a cabo, profesionales intervinientes, plazos aproximados, servicios en que deben prestarse y potenciales necesidades de coordinación y evaluación de resultados, buscando limitar la variabilidad asistencial.

Por otra parte, la indicación de no tratamiento ante aquellas demandas que entendemos que suponen una psicopatologización de los avatares de la vida, no debe significar una confrontación con el "paciente", salvo en aquellos casos en que lo que busca obtener es claros beneficios secundarios (Rendueles, 1992).

Un rechazo hacia él solo supondría su frustración y posiblemente una cronificación de su malestar. El abordaje de tales situaciones precisa una resignificación de la demanda o del problema planteado, tratando de que el paciente cambie la visión que tiene de sus quejas y que deje de entenderlas como algo patológico, para asumirlas como reacciones "normales", que aunque se traduzcan en alteraciones emocionales, estas no tienen el carácter de algo anormal (Ortíz Lobo y Murcia García, 2009).

Este tipo de intervenciones pueden considerarse psicoterapéuticas y tienen un carácter psicopedagógico, conllevando aspectos y secuencias que buscan una reformulación de la demanda de manera que cumpla el objetivo de ayudar al paciente en la resolución de su malestar. 
Estos mismos autores señalan como elementos constituyentes de dicho proceso terapéutico: "una escucha empática, la construcción junto con el sujeto de una versión inicial de lo que le sucede, de lo que espera de nosotros y de la consulta y de cuáles son sus sentimientos al respecto, la deconstrucción de dicha versión y la resignificación de una nueva en la que la problemática se desvincula de una solución sanitaria, y el cierre de la entrevista, en la que el grado de satisfacción del paciente y del profesional medirá la eficacia de la intervención" y proponen una serie de estrategias e intervenciones psicoterapéuticas para llevarlas a cabo, a la vez que plantean los obstáculos, tanto por parte del propio profesional, como del paciente, que más frecuentemente se presentan.

Dichos obstáculos, en el caso de los profesionales, pueden venir derivados de una excesiva identificación con los valores del paciente, o de necesidades personales omnipotentes del terapeuta, o de una orientación profesional en la que lo psicoterapéutico no tenga más límite que la motivación del paciente y no la inexistencia de patología que lo justifique.

A su vez, los obstáculos relacionados con el paciente, pueden venir de actitudes pasivo-dependientes de este, o de que tenga expectativas idealizadas, o por el contrario que en la motivación de sus síntomas existan ganancias secundarias o de otro tipo, siendo en este último caso donde son mayores las resistencias.

Este tipo de intervenciones, de cierta complejidad, precisa de un entrenamiento y tiempo para su aplicación que, en mi opinión, condiciona sus posibilidades de uso en la Atención Primaria en el momento actual, siendo más factible su práctica en el nivel especializado, posiblemente en el contexto de ese procedimiento de evaluación y protocolo de intervenciones que mencioné anteriormente, así como en la recuperación de espacios de trabajo en común entre los dos niveles asistenciales, que permita su aplicación eficaz en el primer nivel.

\section{Conclusiones}

El fenómeno de la medicalización/psicopatologización de la vida va en aumento en los países desarrollados, por la coincidencia de intereses de diversos agentes en ello (sociedad, industria farmacéutica, profesionales sanitarios, etc.), y los conceptos cambiantes del binomio salud/enfermedad, planteando consideraciones de índole cultural, ética, económica, etc.

Existen controversias que derivan de su tipificación (enfermedad/no enfermedad), presentación clínica (subumbral, mixta, comorbilidad), diagnóstico (categorial/dimensional), e indicación o no de tratamiento, pero su morbilidad (16,5\%de la población general padecen problemas de ansiedad-depresión) y su impacto social y ecónómico son muy importantes (en términos en muchos casos de una duración promedio de más de 6 años y la repercusión laboral que produce).

En último extremo es el profesional sanitario (médico/psiquiatra/psicólogo) el que sanciona la demanda como enfermedad/no enfermedad y la indicación o no de tratamiento.

La repercusión de esta demanda en el ámbito asistencial público ha ido creciendo exponencialmente, desbordando la capacidad de respuesta de los equipos (Moré et al., 2008; Ministerio de Sanidad y Consumo, 2007), siendo controvertidos los resultados de su intervención (Hernández Monsalve, 2009; Moré, Huidobro, Rodriguez y Holgado, 2002 ; Moré y Muñoz, 2000), lo que exige que los servicios públicos mejoren la coordinación entre sus niveles asistenciales, así como su organización y la formación de sus profesionales para que puedan intervenir de forma más eficiente y eficaz tanto en su abordaje asistencial, como en una indicación de no tratamiento aceptable para el usuario.

\section{Referencias}

Aiarzaguena, J. M. (2003). Manejo de pacientes con malestar psicosocial en Atención Primaria. Vitoria: Osakidetza- Servicio Vasco de Salud. 
Asociación Española de Neuropsiquiatría (1998). Prestaciones básicas y estándares de calidad en los Servicios de Salud Mental (Coord. Moré, M. A.). Cuaderno Técnico Nº1. Madrid: AEN.

Carmona Calvo, J., Gutierrez Iglesias, A., Maqueda Madrona, T., de Osma Rodriguez, F. y Tejero Bernal, M. (2009). Hacia un modelo de cooperación. En A. Retolaza (Coord.), Trastornos Mentales comunes. Madrid: Asociación Española de Neuropsiquiatría.

Charlton, B. G. (2003). Palliative psycofarmacology: a putative speciality to optimise the subjective quality of life. QJM, An International Journal of Medicine, 96, 375-378.

Consejería de Salud de la Junta de Andalucía (2003). Ansiedad, depresión, somatizaciones: proceso asistencial integrado. Sevilla: Consejería de Salud.

De la Mata, I., Ortiz Lobo, A. (2009). Formulación de casos en salud mental: una guía de entrenamiento. En A. Retolaza, Trastornos Mentales Comunes. Manual de orientación. Madrid: Asociación Española de Neuropsiquiatría.

Ebrahim, S. (2002). The medicalisation of old age. British Medical Journal, 324, 861-863.

Ministerio de Sanidad y Consumo (2007). Estrategia en Salud Mental del Sistema Nacional de Salud. Madrid: Centro de Publicaciones del Ministerio de Salud y Consumo.

Fernandez Liria, A. (2003). Concepto sustantivo y pragmático de la enfermedad mental. En E. Baca y J. Lázaro (Eds.), Hechos y valores en psiquiatría. Madrid: Triacastela.

Gervás, J. y Pérez Fernández, M. (2003). Genética y Prevención cuaternaria. El ejemplo de la hemocromatosis. Atención Primaria, 32(3),158-162.

Goldberg, D. y Huxley, P. (1992). Common Mental Disorders: a Biosocial Model. Londres: Tavistock.

Harrison, J. (2000). Prioritising referrals to a Community Health Team. British Journal of General Practice, 50, 194-198.

Hernández Monsalve, M. (2009). La psicoterapia en el tratamiento de los trastornos mentales comunes. En A. Retolaza, Trastornos mentales comunes. Manual de orientación. Madrid: Asociación Española de Neuropsiquiatría.

Herrán A., Lopez Lanza, J. R., Ganzo, H., Cadinanos, A., Díez Manrique, J.F., Vazquez y Barquero J. (2000). Referral of patients with mental disorders from primary care to mental Elath units. Actas Españolas de Psiquiatría, 28, 13-21

Illich, I. (1999, marzo). L’obsesion de la sante parfaite. Le Monde Diplomatic, pp. 28 y ss.

Osakidetza (2005) Medicalización de la vida. Creación de enfermedades (Editorial). INFAC, Información Farmacoterapéutica de la Comarca. Servicio Central de Publicaciones del Gobierno Vasco.

Kendrick, T. (2000).Why Can’t GPs Follow Guidelines on Depression? British Medical Journal, 320, $200-201$.

Kessler, R., Frank, R., Edlund, M., Kajz, S., Lin, E. y Leaf, P. H. (1997). Differences in the use of psychiatric outpatient services between the United States and Ontario. The New England Journal of Medicine, 336, 551-557.

Kishore, J.A. (2002). Dictionary of Public Health. New Delhi: Century Publications.

Layard, R. (2006). The Depression report: a new deal for depression and anxiety disorders. Londres: Center for Economic Performances. London School of Economics and Political Science. Accesible en htpp://www. cep.lse.ac.uk/research/mentalhealth

Maineti, J.A. (2006). La medicalización de la vida. Electroneurobiologia, 14(3), 71-89.

Márquez, S., Meneu, R. (2003). La medicalización de la vida y sus protagonistas. Gestión Clínica y Sanitaria, 5(2), 47-53.

Meador, C. (1965). The art and science of nondiseases. New England Journal of Medicine, 272, 92-95.

Ministerio de Sanidad y Consumo de España (1986). Ley 14/1986, de 25 de abril, General de Sanidad. Boletín Oficial del Estado ${ }^{\circ} 102$, de 29 de abril.

Mintzes, B. (2002). For and against: Direct to consumer advertising is medicalising normal human experience. British Journal of Medicine, 324, 908-909.

Moré, M. A., Jiménez Arriero, M.A ., Muñoz, P., Muñoz de Morales A., Zufia, J. (2008). Estudio preliminar 
dirigido a la construcción de un cuestionario de derivación de la Atención primaria a los Servicios de Salud Mental. Actas Españolas de Psiquiatría, 32, 210-217.

Moré, M. A. (2009). Propuesta de Curso sobre Valoración e Intervención en los Trastornos mentales comunes en Atención primaria (No publicado). Agencia Laín Entralgo, Madrid.

Moré, M. A. (2007). Gestión del cambio en el proceso de atención a los trastornos adaptativos en un Servicio de Salud Mental. Proyecto Curso Gestión Clínica EADA (No publicado). Barcelona.

Moré, M. A., Huidobro, A., Rodríguez, M., Holgado, P. (2002). Estudio de los abandonos de tratamiento. Archivos de Psiquiatría, 65 (3), 243-262.

Moré, M. A., Muñoz, P.E. (2000). Satisfacción de los usuarios de un Servicio de Salud Mental. Archivos de Psiquiatría, 63(2), 139-157

Morera, J. (1998).Trastornos adaptativos. En J. L.Vázquez Barquero, Psiquiatría en Atención Primaria. Madrid: Aula Médica.

Organización Mundial de la Salud (1948). Constitución de la Organización Mundial de la Salud [Documento online]. Disponible en http://apps.who.int/gb/bd/PDF/bd47/SP/constitucion-sp.pdf

Ortíz Lobo, A. (2008). Los profesionales de salud mental y el tratamiento del malestar. Átopos 26, 26-35.

Ortiz Lobo, A. e Ibáñez Rojo, V. (2012). Prevención cuaternaria en salud mental. En M. Desviat y A. Moreno (Eds.), Acciones de Salud Mental Comunitaria. Principios y Estrategias. Madrid: Asociación Española de Neuropsiquiatría (En prensa).

Ortiz Lobo, A. y Murcia García, L. (2009). La indicación de no tratamiento: aspectos Psicoterapéuticos. En A. Retolaza, Trastornos mentales comunes: Manual de orientación. Madrid: Asociación Española de Neuropsiquiatría.

Orueta Sánchez, R, Santos Rodríguez, C., González Hidalgo, E., Fagundo Becerra E., Alejandre Lázaro, G., Carmona de la Morena J., ... y Butrón Gomez T. (2011). Medicalización de la vida. Revista Clínica de Medicina de Familia, 4(2), 211-218.

Padierna Acero, J., Gastain Saenz, F., Lopez, P. y Etxebeste Anton, A. (1998). La morbilidad Psiquiátrica en Atención primaria: detección y derivación por el médico de familia. Revista de la Asociación Española de Neuropsiquiatría, 24, 21-29.

Rendueles, G. (1992). El usuario gorrón y el terapeuta quemado. Revista de Psiquiatría Pública 3, 115-129.

Rendueles, G. (2009). Construyendo trastornos psiquiátricos: quejicas, simuladores, ventajistas. En A. Retolaza, Trastornos mentales comunes. Manual de orientación. Madrid: Asociación Española de Neuropsiquiatría.

Retolaza, A. (2008). El territorio del malestar. Átopos, 7, 13-26.

Retolaza, A. (2009). La organización asistencial. En Trastornos mentales Comunes. Manual de orientación. Madrid: Asociación Española de Neuropsiquiatría.

Retolaza, A. (2009).Terapéutica. En Trastornos Mentales Comunes. Manual de orientación. Madrid: Asociación Española de Neuropsiquiatría.

Royse, D. (1982). Mental Health needs assesment. Beware of false promises. Community Mental Health Journal, 18, 97-106.

Smith R. (2002). In search of non diseases. British Medical Journal, 324, 883-885.

Tizón, J.L. (2004). Pérdida, pena, duelo: Vivencias, investigación y asistencia. Barcelona: Paidós.

Tizón, J.L., San José, J., Nadal, D. (1997). Protocolos y programas elementales para la Atención primaria a la Salud Mental. Barcelona: Ed. Herder.

Vazquez Barquero, J.L. (1990). Mental Health in Primary care settings. En D. Goldberg y D.Tamtam D (Eds.), The Public Health impact of mental disorders. Toronto: Hogrefe and Huber.

Manuscrito recibido: $11 / 12 / 2012$

Revisión recibida: 12/02/2013

Manuscrito aceptado: 15/02/2013 